\title{
Constructing the System of Mental Health Education in Colleges and Universities Based on Frankel's Logo-therapy
}

\author{
YU Shanshan ${ }^{1, \mathrm{a}}$, LIU Xiaoliang ${ }^{2, \mathrm{~b}}$
}

\author{
${ }^{1}$ Department of Computer Science, Hubei University of Technology, Hongshan, Wuhan, Hubei, China \\ ${ }^{2}$ Department of Civil Engineering, Architecture and Environment, Hubei University of Technology, Hongshan, Wuhan, \\ Hubei, China \\ a2578983679@qq.com \\ b347430401@qq.com
}

\begin{abstract}
Objective: To investigate college students' psychological stress and coping style in the central area during the epidemic of COVID-19 in order to guide the mental health education in colleges and universities based on Frankel's Logo-therapy. Methods: A total of 948 college students from 12 colleges of Hubei University of technology were selected as research objects. The Chinese Perceived Stress Scale and the Simplified Coping Style Questionnaire were used to investigate the students. Results: The college students' overall level of perceived stress was lower than the average, and the frequency of using positive coping style was higher than that of negative coping style. Different groups of college students had different levels of perceived stress and different frequency of adopting positive and negative coping style. There was a positive correlation between perceived stress and positive or negative coping style. $(\mathrm{R}=0.242,0.114, \mathrm{P}<0.001)$. Gender, location, physical condition and total score of perceived stress predicted $8.5 \%$ of positive coping style.Location and total score of perceived stress accounted for $2.1 \%$ of negative coping style. Conclusion: The mental health education in colleges and universities during the epidemic should be more targeted, so as to promote students to adopt the positive coping style and to pursue the significance of their own existence and the realization of their value of life.
\end{abstract}

Keywords: College students, COVID-19 epidemicLogo-therapy, mental health education, stress, coping Style.

\section{基于意义治疗理论的高校心理育人体系构建}

\author{
于姗姗 ${ }^{1, a}$, 刘小亮 ${ }^{2, b}$
}

${ }^{1}$ 湖北工业大学计算机学院, 洪山, 武汉, 湖北, 中国

${ }^{2}$ 湖北工业大学土大建筑与环境学院, 洪山, 武汉, 湖北, 中国

a2578983679@qq.com

b347430401@qq.com

\section{摘要}

目的: 调查新型冠状病毒肺炎疫情下疫情中心城区高校大学生的心理压力及其应对方式，基于弗兰克尔 的意义治疗理论, 为构建重大疫情防控期间的高校心理育人体系提供依据。方法: 采取分层整群抽样的 方法, 选取湖北工业大学 12 个学院共 948 名大学生为研究对象, 用压力知觉量表和简易应对方式问卷进 行调查。结果：大学生整体压力知觉水平中等偏下, 采取积极应对方式的使用频率高于消极应对方式。 不同组别大学生的压力知觉程度和采取积极、消极应对方式的频率不同。压力知觉与积极、消极应对方 
式基本上呈正相关（ $\mathrm{r}$ 值分别为 $0.242 ， 0.114, \mathrm{P}$ 值均 $<0.001$ ）。性别、所在地所属区域、身体状况与压 力知觉总分对积极应对方式有一定的预测意义, 总解释率为 $8.5 \%$; 大学生所在地所属区域与压力知觉总 分对消极应对方式有一定的预测意义，总解释率为 $2.1 \%$ 。结论：重大疫情防控常态化下的高校心理育人 体系更要具针对性, 推动学生通过压力的积极应对, 追寻自身存在的意义和人生价值的实现。

关键词: 大学生; 新冠病毒肺炎疫情; 意义治疗理论; 心理育人；压力；压力应对方式

\section{1. 引言}

新型冠状病毒肺炎疫情自 2020 年初大规模爆 发以来，业已形成常态化防控机制。然而，因其持 续时间长, 涉及范围广, 传染性极强, 并且严重威胁 生命安全, 一定程度上会引发公众的急性应激障碍和 创伤后应急障碍 ${ }^{[1]}$, 也可能带来身处疫情中心城区的 高校大学生的心理健康问题。新型冠状病毒突发不仅 是重大公共卫生事件, 也是重大心理危机事件 [2]。公 共卫生事件突发后, 开展及时有效的心理危机干预是 国家救援体系的重要组成部分 ${ }^{[3]}$ 。对于疫情中心城区 高校、目前仍处于居家学习状态的大学生而言, 及时 排查其心理动态, 并有效开展心理疏导是非常必要的 [4]。

根据压力和健康领域的相关研究显示, 心理健康 问题的主要起因是压力, 而压力主要包括压力源、中 介变量和身心反应三个方面, 其中应对策略是压力源
和身心反应之间最直接, 也是最重要的中介因素之一 [5,6]。所谓应对策略, 是指个体处在一定的压力时期, 针对压力事件所采取的应对措施 ${ }^{[7]}$ 。

基于此, 本研究取样新冠病毒疫情下疫情中心城 区高校的各组别大学生, 试图探究其压力知觉水平和 应对方式的关系; 并通过弗兰克尔的意义治疗理论, 构建重大疫情防控期间的高校心理育人机制。

\section{2. 对象与方法}

\section{1. 被试基本情况}

2019 年 2 月底, 采取分层整群抽样的方法对 湖北工业大学 12 个学院的大学生进行问卷调查, 共发放问卷 1000 份。调查对象利用手机通过在线 答题形式完成调查，剔除无效问卷后，共回收有效 问卷 948 份，有效回收率 94.8\%。被试基本情况见 表 1 .

表 1 被试基本情况统计

\begin{tabular}{cccc}
\hline & 分类 & 人数 & $\%$ \\
\hline \multirow{2}{*}{ 性别 } & 男 & 608 & 64.1 \\
& 女 & 340 & 35.9 \\
\hline \multirow{3}{*}{ 学习阶段 } & 大一年级 & 137 & 14.5 \\
& 大二年级 & 253 & 26.7 \\
& 大三年级 & 416 & 43.9 \\
& 大四年级 & 142 & 15.0 \\
\hline \multirow{4}{*}{ 学科类别 } & 工科类 & 776 & 81.9 \\
& 文科类 & 16 & 1.7 \\
& 理科类 & 111 & 11.7 \\
& 艺术类 & 25 & 2.6 \\
& 管理类 & 20 & 2.1 \\
\hline \multirow{3}{*}{ 政治面貌 } & 中共党员, 含预备党员 & 107 & 11.3 \\
& 共青团员 & 789 & 83.2 \\
& 群众 & 52 & 5.5 \\
\hline \multirow{3}{*}{ 目前所在地 } & 武汉市内 & 150 & 15.8 \\
& 武汉市外湖北省内 & 605 & 63.8
\end{tabular}




\begin{tabular}{cccc} 
& $\begin{array}{c}\text { 湖北省外国内 } \\
\text { 国外 }\end{array}$ & 192 & 20.3 \\
& 城市 & 1 & 0.1 \\
\hline 所在地所属 & 近郊 & 423 & 44.6 \\
区域 & 农村 & 119 & 12.6 \\
& 有 & 406 & 42.8 \\
\hline 有无直接接 & 无 & 10 & 1.1 \\
触新冠病毒 & 不确定 & 915 & 96.5 \\
确诊患者 & 非常健康 & 23 & 2.4 \\
\hline \multirow{3}{*}{ 目前的身体 } & 良好 & 790 & 83.3 \\
状况 & 一般 & 140 & 14.8 \\
& 较差 & 17 & 1.8 \\
\hline
\end{tabular}

\section{2. 研究工具}

\section{2. 1. 基本情况调查表}

自制调查表, 收集被测的社会人口学信息, 维度 包括性别、学习阶段、学科类别、政治面貌、目前所 在地、所在地所属区域、有无直接接触新冠病毒确诊 患者、自我判定的身体状况等。

\subsection{2. 压力知觉量表 (Chinese 14-item PSS)}

基于杨廷忠修订的中文版量表，测评个体面对日 常琐事、重大事件和应激源的改变这三种压力情境, 对生活压力感知的程度。量表包括紧张感和失控感两 个维度, 共由 14 个条目组成, 采用 5 点计分的方式, 其中 7 项正向计分, 7 项反向计分。本研究中总量表 Cronbach's $\alpha$ 系数为 0.82 ; 各维度 Cronbach's $\alpha$ 系数为 0.793-0.856.

\subsection{3. 简易应对方式问卷 (Simplified Coping Style Questionnaire, SCSQ)}

用来评估个体遭受困难或挫折时所采取的态度 和做法。量表包括积极应对方式（第 1-12 项）和消 极应对方式 (第 13-20 项) 两个维度, 共由 20 个条 目组成, 采用 0-3 分四级评分法, 即从不采取、偶尔、 有时、经常采取。本研究总量表 Cronbach's $\alpha$ 系数为 0.972 , 各维度 Cronbach's $\alpha$ 系数为 $0.969-0.972$ 。

\section{3. 统计方法}

采用 Excel 录入数据, 导入 SPSS20.0, 对大学生 的基本信息、压力知觉总分、紧张感、失控感、积极 应对方式和消极应对方式进行频率分析、相关分析和 回归分析等。首先，对各变量进行描述性统计分析、 独立样本 $\mathrm{t}$ 检验和单因素方差分析; 进而, 采用 Pearson 相关分析检验变量之间的相关性; 最后, 在 变量有显著相关的基础上, 分别以积极应对方式和消 极应对方式为因变量, 以性别、所在地所属区域、身
体状况与压力知觉为自变量, 设置亚变量后, 采用分 层回归分析, 进一步研究各自变量对大学生压力应对 方式的影响, 检验水准 $\alpha=0.05$.

\section{3. 结果}

\section{1. 压力知觉和应对方式的现状}

结果显示, 紧张感和失控感均值小于 21 分, 压 力知觉均值小于 42 分, 题目均值小于 3 分。由于压 力知觉量表采用 5 点计分法, 中数为 3 , 可以说疫情 中心城区高校的大学生压力知觉水平处于中等偏下。 而就大学生压力应对现状的分析结果, 积极应对方式 的均值远高于消极应对方式, 说明了大学生积极应对 方式的使用频率高于消极应对方式。

表 2 大学生压力知觉和应对方式的现状

\begin{tabular}{cccc}
\hline & 最小值 & 最大值 & $\bar{x} \pm \mathrm{s}$ \\
\hline 压力知觉总分 & 14 & 70 & $39.3597 \pm 7.84945$ \\
紧张感 & 7 & 35 & $18.9367 \pm 4.15642$ \\
失控感 & 7 & 35 & $20.423 \pm 4.58331$ \\
积极应对方式 & 0 & 36 & $21.6108 \pm 8.06556$ \\
消极应对方式 & 0 & 24 & $9.7627 \pm 5.14947$ \\
\hline
\end{tabular}

\section{2. 不同组别大学生的压力知觉、积极应对方 式和消极应对方式的情况比较}

研究发现, 除消极应对方式维度外, 各项得分在 性别上都存在显著差异，女性的均值均明显高于男 性, 即女性压力知觉水平较高, 应对较为频繁。不同 政治面貌个体的紧张感有显著差异，中共党员（含预 备党员）的紧张感高于共青团员和群众。身处不同区 域个体的积极应对方式和消极应对方式有显著差异, 在城市的大学生积极应对和消极应对方式的均值都 高于近郊和农村。个体自我判定的身体状况不同，在 紧张感上有极其显著的统计学差异, 在积极应对方式 
上也存在显著差异。另外, 学习阶段、学科类别、目 前所在地、有无直接接触新冠病毒确诊患者在压力知 觉、紧张感、失控感、积极应对方式和消极应对方式
各维度的得分比较上都没有显著差异（ $\mathrm{P}>0.05 ） 。$ 见表 3。

表 3 大学生压力知觉、积极和消极应对方式在不同组别的比较

\begin{tabular}{|c|c|c|c|c|c|c|}
\hline \multirow[t]{2}{*}{ 项目 } & \multirow[t]{2}{*}{ 类别 } & $\begin{array}{l}\text { 压力知觉 } \\
\text { 总分 }\end{array}$ & 紧张感 & 失控感 & $\begin{array}{c}\text { 积极应对 } \\
\text { 方式 }\end{array}$ & $\begin{array}{c}\text { 消极应对 } \\
\text { 方式 }\end{array}$ \\
\hline & & $\bar{x} \pm \mathrm{s}$ & $\bar{x} \pm \mathrm{s}$ & $\bar{x} \pm \mathrm{s}$ & $\bar{x} \pm \mathrm{s}$ & $\bar{x} \pm \mathrm{s}$ \\
\hline \multirow{4}{*}{ 性别 } & 男 & $38.5214 \pm 8.56814$ & $18.3503 \pm 4.04540$ & $20.1711 \pm 5.00020$ & $20.7862 \pm 8.55857$ & $9.7155 \pm 5.44764$ \\
\hline & 女 & $40.8588 \pm 6.09541$ & $19.9853 \pm 3.43371$ & $20.8735 \pm 3.68748$ & $23.0853 \pm 6.86802$ & $9.8471 \pm 4.57454$ \\
\hline & $\mathrm{t}$ & -4.874 & -6.335 & -2.467 & -4.516 & -0.396 \\
\hline & $\mathrm{P}$ & $0.000 * * *$ & $0.000 * * *$ & $0.014 * *$ & $0.000 * * *$ & 0.692 \\
\hline \multirow{6}{*}{$\begin{array}{l}\text { 学习阶 } \\
\text { 段 }\end{array}$} & 大一年级 & $39.8248 \pm 7.19773$ & $19.365 \pm 3.88033$ & $20.4599 \pm 4.51308$ & $21.8686 \pm 8.00351$ & $9.5182 \pm 5.12649$ \\
\hline & 大二年级 & $38.9723 \pm 7.38102$ & $18.747 \pm 3.83582$ & $20.2253 \pm 4.28830$ & $21.8814 \pm 7.74096$ & $10.0237 \pm 5.01896$ \\
\hline & 大三年级 & $39.1563 \pm 8.70796$ & $18.8077 \pm 4.61548$ & $20.3486 \pm 4.91383$ & $21.2115 \pm 8.43335$ & $9.8726 \pm 5.33013$ \\
\hline & 大四年级 & $40.1972 \pm 6.47991$ & $19.2394 \pm 3.48234$ & $20.9577 \pm 4.13920$ & $22.0493 \pm 7.60583$ & $9.2113 \pm 4.85657$ \\
\hline & F & 0.998 & 1.045 & 0.84 & 0.621 & 0.925 \\
\hline & $\mathrm{P}$ & 0.393 & 0.372 & 0.472 & 0.602 & 0.428 \\
\hline \multirow{7}{*}{$\begin{array}{c}\text { 学科类 } \\
\text { 别 }\end{array}$} & 工科类 & $39.3299 \pm 7.94948$ & $18.8956 \pm 4.16971$ & $20.4343 \pm 4.68335$ & $21.5966 \pm 8.18644$ & $9.6688 \pm 5.17761$ \\
\hline & 文科类 & $41.4375 \pm 4.39649$ & $20.3125 \pm 3.04891$ & $21.1250 \pm 3.18067$ & $23.2500 \pm 8.11172$ & $9.4375 \pm 4.41163$ \\
\hline & 理科类 & $38.7838 \pm 7.93199$ & $18.4955 \pm 4.13604$ & $20.2883 \pm 4.46682$ & $21.1802 \pm 7.76378$ & $9.9730 \pm 5.09627$ \\
\hline & 艺术类 & $40.2400 \pm 6.28676$ & $20.5200 \pm 3.53695$ & $19.7200 \pm 3.38526$ & $23.0800 \pm 5.69298$ & $12.3600 \pm 5.13874$ \\
\hline & 管理类 & $40.9500 \pm 7.38758$ & $19.9000 \pm 4.75616$ & $21.0500 \pm 3.57587$ & $21.4000 \pm 7.78933$ & $9.2500 \pm 4.38748$ \\
\hline & $\mathrm{F}$ & 0.715 & 1.953 & 0.359 & 0.455 & 1.772 \\
\hline & $\mathrm{P}$ & 0.581 & 0.1 & 0.838 & 0.769 & 0.132 \\
\hline \multirow{5}{*}{$\begin{array}{l}\text { 政治面 } \\
\text { 貌 }\end{array}$} & $\begin{array}{c}\text { 中共党员 } \\
\text { (含预备党员) }\end{array}$ & $40.9907 \pm 6.83705$ & $19.9065 \pm 3.88401$ & $21.0841 \pm 4.03083$ & $22.3271 \pm 8.67416$ & $8.8785 \pm 4.99568$ \\
\hline & 共青团员 & $39.1572 \pm 7.81273$ & $18.8150 \pm 4.12141$ & $20.3422 \pm 4.58252$ & $21.5501 \pm 7.93672$ & $9.8542 \pm 5.12957$ \\
\hline & 群众 & $39.0769 \pm 9.87539$ & $18.7885 \pm 4.97577$ & $20.2885 \pm 5.54955$ & $21.0577 \pm 8.76583$ & $10.1923 \pm 5.65699$ \\
\hline & $\mathrm{F}$ & 2.615 & 3.3 & 1.259 & 0.566 & 1.887 \\
\hline & $\mathrm{P}$ & 0.074 & $0.037 *$ & 0.284 & 0.568 & 0.152 \\
\hline \multirow{6}{*}{$\begin{array}{l}\text { 目前所 } \\
\text { 在地 }\end{array}$} & 武汉市内 & $39.2800 \pm 9.02057$ & $19.0800 \pm 4.78605$ & $20.2000 \pm 5.04344$ & $21.3200 \pm 8.06128$ & $9.5667 \pm 5.52653$ \\
\hline & $\begin{array}{l}\text { 武汉市外 } \\
\text { 湖北省内 }\end{array}$ & $39.2760 \pm 7.57445$ & $18.8281 \pm 3.96385$ & $20.4479 \pm 4.52579$ & $21.3802 \pm 8.07422$ & $9.5686 \pm 5.05866$ \\
\hline & $\begin{array}{c}\text { 湖北省外 } \\
\text { 国内 }\end{array}$ & $39.6771 \pm 7.77430$ & $19.1615 \pm 4.24140$ & $20.5156 \pm 4.41437$ & $22.5729 \pm 8.03167$ & $10.526 \pm 5.09793$ \\
\hline & 国外 & 41 & 20 & 21 & 20 & 10 \\
\hline & $\mathrm{F}$ & 0.147 & 0.405 & 0.155 & 1.154 & 1.77 \\
\hline & $\mathrm{P}$ & 0.932 & 0.749 & 0.926 & 0.326 & 0.151 \\
\hline \multirow{5}{*}{$\begin{array}{c}\text { 所在地 } \\
\text { 所属区 } \\
\text { 域 }\end{array}$} & 城市 & $39.4823 \pm 7.99372$ & $18.9976 \pm 4.22557$ & $20.4846 \pm 4.74358$ & $22.4515 \pm 7.82946$ & $10.2742 \pm 5.13611$ \\
\hline & 近郊 & $38.1008 \pm 9.18584$ & $18.3866 \pm 4.64158$ & $19.7143 \pm 5.24508$ & $20.9496 \pm 7.13175$ & $9.4118 \pm 4.68755$ \\
\hline & 农村 & $39.6010 \pm 7.23629$ & $19.0345 \pm 3.92570$ & $20.5665 \pm 4.18159$ & $20.9286 \pm 8.49036$ & $9.3325 \pm 5.25548$ \\
\hline & $\mathrm{F}$ & 1.777 & 1.201 & 1.662 & 4.178 & 3.802 \\
\hline & $\mathrm{P}$ & 0.17 & 0.301 & 0.19 & $0.016^{*}$ & $0.023^{*}$ \\
\hline \multirow{5}{*}{$\begin{array}{c}\text { 有无直 } \\
\text { 接接触 } \\
\text { 新冠病 } \\
\text { 毒确诊 } \\
\text { 患者 }\end{array}$} & 有 & $40.9000 \pm 7.20262$ & $19 \pm 4.94413$ & $21.9 \pm 4.14863$ & $22.1 \pm 8.77433$ & $9.3 \pm 2.71006$ \\
\hline & 无 & $39.3825 \pm 7.81698$ & $18.9399 \pm 4.13314$ & $20.4426 \pm 4.57179$ & $21.6262 \pm 8.05018$ & $9.7421 \pm 5.13101$ \\
\hline & 不确定 & $37.7826 \pm 9.41448$ & $18.7826 \pm 4.88929$ & $19 \pm 5.07221$ & $20.7826 \pm 8.69146$ & $10.7826 \pm 6.59171$ \\
\hline & $\mathrm{F}$ & 0.66 & 0.017 & 1.638 & 0.141 & 0.498 \\
\hline & $\mathrm{P}$ & 0.517 & 0.983 & 0.195 & 0.868 & 0.608 \\
\hline \multirow{6}{*}{$\begin{array}{l}\text { 自我判 } \\
\text { 定的身 } \\
\text { 体状况 }\end{array}$} & 非常健康 & $39.3367 \pm 7.99147$ & $18.7949 \pm 4.15705$ & $20.5418 \pm 4.69583$ & $21.9278 \pm 8.19571$ & $9.7392 \pm 5.26247$ \\
\hline & 良好 & $39.1643 \pm 7.33339$ & $19.3429 \pm 4.09030$ & $19.8214 \pm 4.06117$ & $20.2857 \pm 7.32030$ & $9.6214 \pm 4.49695$ \\
\hline & 一般 & $42.1176 \pm 4.66211$ & $22.0588 \pm 3.45454$ & $20.0588 \pm 2.81670$ & $17.9412 \pm 6.11832$ & $11.9412 \pm 4.77586$ \\
\hline & 较差 & 38 & 21 & 17 & 19 & 11 \\
\hline & $\mathrm{F}$ & 0.74 & 4.071 & 1.203 & 2.892 & 1.074 \\
\hline & $\mathrm{P}$ & 0.528 & $0.007 * *$ & 0.307 & $0.034 *$ & 0.359 \\
\hline
\end{tabular}




\section{3. 大学生压力知觉与积极、消极应对方式的 相关分析}

除失控感和消极应对方式无显著相关，其他各项 都有极其显著的统计学关系, 呈正相关 ( P 值均 <0.001）。压力知觉总分与积极应对方式、消极应对 方式的 $\mathrm{r}$ 值为 0.242 和 0.114 ; 紧张感与积极应对方式、 消极应对方式的 $\mathrm{r}$ 值为 0.124 和 0.153 ; 失控感和积极 应对方式的 $r$ 值为 0.302 。见表 4 。

表 4 大学生压力知觉与积极、消极应对方式的相关 ( $\mathrm{r}$ 值)

\begin{tabular}{lll}
\hline & 积极应对方式 & 消极应对方式 \\
\hline 压力知觉总分 & $0.242 * * *$ & $0.114 * * *$ \\
紧张感 & $0.124 * * *$ & $0.153 * * *$ \\
失控感 & $0.302 * * *$ & 0.057 \\
\hline
\end{tabular}

\section{4. 大学生性别、所在地所属区域、身体状况 与压力知觉对于积极应对方式的回归分析 ${ }^{[8]}$}

就表 5 可知, 控制变量中性别与积极应对方式具
有极其显著的相关（ $\mathrm{P}<0.001 ）$ ，所在地所属区域、 自我判定的身体状况与积极应对方式显著相关 $(\mathrm{P}<0.05)$; 因此将其纳入最终的回归方程中，对数 据进行分层回归。在回归分析前, 将性别、所在地所 属区域、身体状况设置为哑变量, 同时对压力知觉进 行中心化处理。在第 1 层纳入控制变量性别, 第 2 层 纳入所在地所属区域, 第 3 层纳入自我判定的身体状 况, 第 4 层纳入中心化的压力知觉总分。就表 5 的第 1 步显示, 性别对积极应对方式有预测作用 $(\beta=-0.137, P<0.001)$; 第 2 步显示, 所在地所属区 域中只有城市这一维度具有预测作用 $(\beta=-0.087$, $\mathrm{P}<0.01)$ ，近郊和农村均无明显预测作用（ $\mathrm{P}>0.05 ）$;

第 3 步显示, 自我判定的身体状况中非常健康这 一维度有预测作用（ $\beta=0.091 ， P<0.01 ）$ ，良好、一般、 较差均无明显预测作用（ $\mathrm{P}>0.05 ）$; 第 4 步显示, 压 力知觉总分也有预测作用 $(\beta=0.227, \mathrm{P}<0.001)$ 。回 归方程控制性别这一变量, 纳入所在地所属区域、自 我判定的身体状况 2 个变量后, $\triangle \mathrm{R} 2$ 值分别为 0.008 、 $0.008, \triangle \mathrm{F}$ 值具有统计学意义, 分别为 $7.328 、 8.174$ $(\mathrm{P}<0.01)$ 。回归方程纳入压力知觉总分后, 方程的 $\triangle R 2$ 值为 $0.051, \triangle F$ 值为 $52.086 （ \mathrm{P}<0.001)$ 。在回 归方程中, 性别、所在地所属区域、身体状况与压力 知觉对于积极应对方式总的解释率为 $8.5 \%$ 。

表 5 大学生性别、所在地所属区域、身体状况与压力知觉对于积极应对方式的分层回归分析

\begin{tabular}{|c|c|c|c|c|c|c|c|c|c|c|c|c|}
\hline \multirow[b]{2}{*}{ 自变量 } & \multicolumn{3}{|c|}{ 第 1 步 } & \multicolumn{3}{|c|}{ 第 2 步 } & \multicolumn{3}{|c|}{ 第 3 步 } & \multicolumn{3}{|c|}{ 第 4 步 } \\
\hline & $\mathrm{B}$ 值 & 标准误差 & $\beta$ 值 & $\mathrm{B}$ 值 & 标准误差 & $\beta$ 值 & B 值 & 标准误差 & $\beta$ 值 & B 值 & $\begin{array}{l}\text { 标准误 } \\
\text { 差 }\end{array}$ & $\beta$ 值 \\
\hline 性别 & -2.299 & 0.541 & $-0.137 * * *$ & -2.226 & 0.54 & $-0.132 * * *$ & -2.275 & 0.538 & $-0.135 * * *$ & -1.731 & 0.53 & $-0.103 * * *$ \\
\hline $\begin{array}{c}\text { 所在地所属区 } \\
\text { 域: 城市 } \\
\text { 自我判定的身 } \\
\text { 体状况: 非常健 } \\
\text { 康 }\end{array}$ & & & & 1.411 & 0.521 & $0.087^{* *}$ & 1.396 & 0.519 & $0.086^{* *}$ & 1.989 & 0.506 & $-0.085 * *$ \\
\hline 压力知觉总分 & & & & & & & & & & 0.233 & 0.032 & $0.227 * * *$ \\
\hline R2 值 & & 0.019 & & & 0.026 & & & 0.035 & & & 0.085 & \\
\hline$\triangle \mathrm{R} 2$ 值 & & & & & 0.008 & & & 0.008 & & & 0.051 & \\
\hline $\mathrm{F}$ 值 & & 18.037 *** & & & & & & & & & & \\
\hline$\Delta \mathrm{F}$ 值 & & & & & $7.328 * *$ & & & $8.174 * *$ & & & $52.086^{* *}$ & \\
\hline
\end{tabular}

表 6 大学生所在地所属区域与压力知觉对于消极应对方式的分层回归分析

\begin{tabular}{|c|c|c|c|c|c|c|}
\hline \multirow{2}{*}{ 自变量 } & \multicolumn{3}{|c|}{ 第 1 步 } & \multicolumn{3}{|c|}{ 第 2 步 } \\
\hline & B 值 & 标准误差 & $\beta$ 值 & B 值 & 标准误差 & $\beta$ 值 \\
\hline 所在地所属区域: 城市 & 0.924 & 0.335 & $0.089^{* *}$ & 0.907 & 0.333 & $0.088^{* *}$ \\
\hline 压力知觉总分 & & & & 0.074 & 0.021 & $0.113^{* * *}$ \\
\hline R2 值 & \multicolumn{3}{|c|}{0.008} & \multicolumn{3}{|c|}{0.021} \\
\hline$\triangle \mathrm{R} 2$ 值 & & & & \multicolumn{3}{|c|}{0.013} \\
\hline $\mathrm{F}$ 值 & & $7.591 * *$ & & & & \\
\hline$\Delta \mathrm{F}$ 值 & & & & \multicolumn{3}{|c|}{$12.351^{* * *}$} \\
\hline
\end{tabular}




\section{5. 大学生所在地所属区域与压力知觉对于 消极应对方式的回归分析 ${ }^{[9]}$}

就表 6 可知, 控制变量中所在地所属区域与消极 应对方式显著相关 $(\mathrm{P}<0.05)$; 因此将其纳入最终的 回归方程中, 对数据进行分层回归。在回归分析前, 将所在地所属区域设置为亚变量, 同时对压力知觉进 行中心化处理。在第 1 层纳入所在地所属区域, 第 2 层纳入中心化的压力知觉总分。就表 6 的第 1 步显示, 所在地所属区域中只有城市这一维度对消极应对方 式有预测作用 $(\beta=0.089, P<0.01)$, 近郊和农村均无 明显预测作用 $(P>0.05)$; 第 2 步显示, 压力知觉总 分也有预测作用 $(\beta=0.113, P<0.001)$ 。回归方程控 制所在地所属区域这一变量, 纳入压力知觉总分后, $\triangle R 2$ 值为 $0.013, \triangle F$ 值具有统计学意义, 为 12.351 $(\mathrm{P}<0.001)$ 。在回归方程中, 所在地所属区域的城 市维度与压力知觉对于消极应对方式总的解释率为 $2.1 \%$ 。

\section{4. 结论}

如前所述, 大学生整体压力知觉水平处于中等偏 下水平, 采取积极应对方式的使用频率高于消极应对 方式。以下因素可能影响到新冠病毒疫情期间大学生 的压力水平和压力应对方式: 第一, 尽管武汉市是疫 情爆发的中心地带, 但疫情相关信息扩散和封城等措 施开始执行的时间是在寒假, 某些离开武汉的大学生 感受到的压力不是很大。第二, 社会各界和高校都采 取了一系列切实有效的疫情防控措施, 构建疫情防控 云平台, 高度关注疫情期间的心理疏导和教育引导。 第三, 已有研究表明, 高认知者有明显的低抑有特征 [10], 大学生对新冠肺炎疫情的认知越全面, 预防措施 了解得越到位, 其心理状态越好, 越能积极应对疫情 [11]。作为知识分子的大学生, 往往具有一定的信息篮 选和理性评判能力, 更容易迅速接受和掌握疫情相关 的科学知识, 对疫情防控阻击战的胜利满怀信心。总 之, 疫情期间大学生群体面对的压力处于一个可接受 的、不会对其造成较大影响的水平 ${ }^{[12]}$ 。

其中, 不同性别大一新生在压力知觉总体、紧张 感和失控感维度均存在显著差异, 女生比男生更容易 体会到紧张和失控。女性更易受到环境应激源的影响 [13]。2003 年非典期间有性别差异研究显示: 在主观 反应中, 男性的负面情绪联想高于女性; 女性的负面 判断联想高于男性 ${ }^{[14]}$ 。这可能的原因是, 传统的性别 模型把男性描述为具有攻击性的、独立的、非情感化 的、控制的; 女性是被动、依赖和富于爱心、情感化 的 ${ }^{[15]}$ 。男女生的认知方式不同, 女性情感更加细淢, 更容易产生紧张与失控的情绪。中共党员（含预备党 员) 的紧张感高于共青团员和群众, 体现出更多的责 任与担当; 因此要提高疫情防控工作的政治站位, 引 导学生自我教育 ${ }^{[16]}$ 。身处城市的大学生容易接受到更 多的防控信息, 其积极应对和消极应对方式程度均高 于近郊和农村。自我判定身体情况较差的大学生, 紧
张感会更强。高校在组织心理疏导活动时, 除关照群 体心理和行为外, 更要重视并开展具有针对性的个体 学生干预。

除失控感和消极应对方式无显著相关外, 大学生 的压力知觉与积极、消极应对方式均呈现正相关。值 得注意的是, 性别、所在地所属区域、身体状况与压 力知觉对于积极应对方式; 以及大学生所在地所属区 域与压力知觉对于消极应对方式都有一定的解释度, 对于高校重大疫情防控期间的心理育人具有预测和 指导意义。

20 世纪中叶, 奥地利著名精神医学家和心理学 家维克多. 弗兰克尔结合自身在德国纳粹集中营的苦 难经历及其长期的医疗实践形成了意义治疗理论, 即, “对照弗洛伊德精神分析所着重的唯乐原则（或 可称作唯乐意志), 和对照阿德勒心理学派所着重的 并称之为 '争取优胜' 的权力意志, 提出了意义意 志”[17]。他认为尽管人都要受到生物、心理和社会等 多重因素的制约，但 “这种制约并不能从根本上决定 个人对其生活情境的反应。无论是处于什么条件甚至 是在几乎无能为力的状态下, 人也会站在自己的立场 上来进行选择、战胜外界环境所施加的限制, 实现自 己的理想和心愿, 这就说明人类完全具有精神上的自 由。"[18]

疫情防控常态化下的高校心理育人同样致力于 面对重大负性事件的疏导、干预和调节，可以在该理 论的指导下，注重发展人的精神自由，聚焦于“人存 在的意义以及人对此意义的追寻上”，针对不同组别 学生的特征, 帮助他们分析自身存在的意义, 使其精 神因素复苏, 从而全面地认识自己的自由和责任。[19] 通过群体辅导和个体咨询, 引导学生尽量避免消极应 对方式, 主动选择积极应对方式: 通过脚踏实地的学 习与工作, 提升自我成就感, 实现创造性的价值; 体 悟亲人、朋友乃至全社会的支持与关爱，同时感受大 自然的美好, 把爱内化为有意识的行为, 实现经验性 的价值; 对于不可更改的命运，适当改变自己看待事 物的视角, 重新认识生命中哪些事物是真正重要的, 确立态度性的价值; 最终通过重大疫情的应对, 促进 自我同一性的良性发展 ${ }^{[20]}$ 。

\section{项目基金}

本文为高校思想政治工作队伍培训研修中心 (北 京师范大学) 开放课题《重大应急突发事件下大学生 群体创伤后成长研究》的阶段性成果之一。

\section{REFERENCES}

[1] Chang,J.H.,Yuan,Y.X.,Wang,D.(2020) Analysis of mental health status and influencing factors of college students with novel coronavirus pneumonia.Journal of Southern Medical University,40(2):171-176.

[2] Fu,G.S.,Wang,M.(2020)Construction of 
psychological crisis intervention mechanism in colleges and universities under the epidemic situation of new coronavirus pneumonia.Monthly Journal of Psychology,4(15):26-28.

[3] Sun,H.W.,Cheng,X.L.,Wang,Y.Y.,et al.(2018)Construction of psychological crisis intervention system for public health emergencies in China.Chinese Journal of Hygiene Rescue(Electronic Editio),4(3): 141-144.

[4] Zhang,X.H.,Ye,T.T.,Yao,L.J.,et al.(2020)Investigation on the psychological behavior of medical students during the outbreak of novel coronavirus pneumonia (Covid-19).Journal of Tropical Medicine,20(5):576-580.

[5] Lazarus RS.(1993)From psychological stress to the emotions:A history of changing outlooks.Ann Rev Psychol,44:2-19.

[6] Coyne JC.(1991)Social factors and Psychopathology:Stress,social support and coping process.Ann Rev Psychol,42:401-425.

[7] Xv,F.M.,Yv,P.,Feng,H.(2004)A study on the mental health of college students in Tianjin during the SARS epidemic period.Chinese Journal of School Health,8(4):398-400.

[8][9] Duan,Z.Y.,Yang,X.F.,Liu,J.(2001)Effects of gender life expenditure and time management on network dependence of freshmen.Chinese Journal of School Health,41(1):130-133.

[10] Tong,H.J.(2004)Stress response pattern and characteristics of SARS.Acta Psychologica Sinica, 36(1):103-109.

[11] Sun,Z.Q.(2019)Application of positive psychology in mental health education of college students.Journal of Zaozhuang University,36(3):131-135.

[12] Yin,G.E.,Xv,F.M.,Yv,P.,et al.(2003)Study on the pressure source and pressure of college students during the period of SARS epidemic.Studies of Psychology and Behavior,1(3):236-239.

[13] Skaff, M.M., J.W. Finney and R.H. Moos,(1999) Gender differences in problem drinking and depression: different "vulnerabilities?".American journal of community psychology,27(1):25-54.

[14] Research Group on psychological meaning of SARS.(2004)Gender comparison of psychological meaning of SARS.Collection of Women's Studies, 1(1):23-27.

[15] Wang,J.J,Jia,X.M.(2004)Heterosexual intercourse and gender role cognition of college students.Journal of Beijing Institute of Technology
(Social Sciences Edition) ,12(6):44-46.

[16] Yang,T.(2020)Ways and means of promoting the maturity and growth of college students in coping with major epidemic.Theory Research,1:1-3.

[17] Frank.V.E.,Sang J.P.(1994) Man's Search For Meaning.China Translation \& Publishing Corporation, Beijing.

[18] Jia,L.X.(2004)An analysis of Frankl's theory of meaningful therapy.Medicine \&amp; Philosophy,(8):73-74.

[19] Gould W.B., ChangX.L., et al.(2000)Viktor E. Frankel: Life with Meaning.China Light Industry Press, Beijing.

[20] Li,B.,Li,L.Y.,An,Q.,et al.(2005)The influence of major crisis life events on college students psychological growth -- the influence of SARS on college students self-identity.China Journal of Health Psychology,13(1):48-50. 